\title{
BMJ Open Assessing the effectiveness of 3 months day and night home closed-loop insulin delivery in adults with suboptimally controlled type 1 diabetes: a randomised crossover study protocol
}

\author{
Lalantha Leelarathna, ${ }^{1}$ Sibylle Dellweg, ${ }^{2}$ Julia K Mader ${ }^{3}$ Katharine Barnard, ${ }^{4}$ \\ Carsten Benesch, ${ }^{2}$ Martin Ellmerer, ${ }^{3}$ Lutz Heinemann, ${ }^{2}$ Harald Kojzar, ${ }^{3}$ \\ Hood Thabit, ${ }^{1}$ Malgorzata E Wilinska, ${ }^{1}$ Tim Wysocki, ${ }^{5}$ Thomas R Pieber, ${ }^{3}$ \\ Sabine Arnolds, ${ }^{2}$ Mark L Evans, ${ }^{1}$ Roman Hovorka, ${ }^{1}$ On behalf of the AP@ home \\ consortium
}

To cite: Leelarathna L, Dellweg S, Mader JK, et al. Assessing the effectiveness of 3 months day and night home closed-loop insulin delivery in adults with suboptimally controlled type 1 diabetes: a randomised crossover study protocol. BMJ Open 2014;4: e006075. doi:10.1136/ bmjopen-2014-006075

- Prepublication history and additional material is available. To view please visit the journal (http://dx.doi.org/ 10.1136/bmjopen-2014006075).

Received 9 July 2014 Accepted 15 August 2014

CrossMark

For numbered affiliations see end of article.

Correspondence to Dr Roman Hovorka; rh347@cam.ac.uk

\section{ABSTRACT}

Introduction: Despite therapeutic advances, many people with type 1 diabetes are still unable to achieve optimal glycaemic control, limited by the occurrence of hypoglycaemia. The objective of the present study is to determine the effectiveness of day and night home closed-loop over the medium term compared with sensor-augmented pump therapy in adults with type 1 diabetes and suboptimal glycaemic control.

Methods and analysis: The study will adopt an open label, three-centre, multinational, randomised, two-period crossover study design comparing automated closed-loop glucose control with sensor augmented insulin pump therapy. The study will aim for 30 completed participants. Eligible participants will be adults ( $\geq 18$ years) with type 1 diabetes treated with insulin pump therapy and suboptimal glycaemic control (glycated haemoglobin $(\mathrm{HbA} 1 \mathrm{c}) \geq 7.5 \%$ $(58 \mathrm{mmol} / \mathrm{mmol})$ and $\leq 10 \%(86 \mathrm{mmol} / \mathrm{mmol}))$. Following a 4-week optimisation period, participants will undergo a 3-month use of automated closed-loop insulin delivery and sensor-augmented pump therapy, with a 4-6 week washout period in between. The order of the interventions will be random. All analysis will be conducted on an intention to treat basis. The primary outcome is the time spent in the target glucose range from 3.9 to $10.0 \mathrm{mmol} / \mathrm{L}$ based on continuous glucose monitoring levels during the 3 months free living phase. Secondary outcomes include HbA1c changes; mean glucose and time spent above and below target glucose levels. Further, participants will be invited at baseline, midpoint and study end to participate in semistructured interviews and complete questionnaires to explore usability and acceptance of the technology, impact on quality of life and fear of hypoglycaemia.

Ethics and dissemination: Ethical approval has been obtained at all sites. Before screening, all participants will be provided with oral and written information about the trial. The study will be disseminated by peer-review publications and

\section{Strengths and limitations of this study}

The study is a three-centre, multinational, randomised, two-period crossover study comparing automated closed-loop glucose control with sensor augmented insulin pump therapy.

- The study duration is 3 months and is the longest day and night closed-loop study under complete free living conditions to be conducted.

- All participants will undergo a period of structured treatment optimisation prior to the study intervention and receive equal attention from study team during each study arm.

- Psychosocial evaluation using semistructured interviews and validated questionnaires will be conducted to determine the acceptability of intervention, quality of life, diabetes self-management and fear of hypoglycaemia.

- The study is open label and aim for $30 \mathrm{com}$ pleted participants.

conference presentations.

Trial registration number: NCT01961622

(ClinicalTrials.gov).

\section{INTRODUCTION}

Type 1 diabetes mellitus (T1D) is characterised by an absolute deficiency of insulin caused by immunologically-mediated damage to the $\beta$ cells in the pancreas and raised blood glucose levels. It is one of the commonest endocrine and metabolic conditions in children and adults. It is estimated that approximately 285 million adults (5-15\% T1D) and 480000 children (95\% T1D) 
worldwide suffer from diabetes. ${ }^{1}$ Recent reports suggest that the incidence and prevalence of T1D are increasing in many countries, at least in the under 15-year age group with the predicted number of new cases of childhood diabetes in Europe increasing to 24400 in 2020 from 15000 in $2005 .^{23}$

Despite the rapid advancements in insulin pump technology and the ongoing development of more physiological insulin preparations, achieving optimal glycaemic control while avoiding hypoglycaemia ${ }^{4}$ remains a challenge for many people with T1D. ${ }^{5}{ }^{6}$ The emergence of continuous glucose monitoring (CGM) over the last decade, which enables users to view real-time interstitial glucose readings and receive alarms for impending hypoglycaemia or hyperglycaemia, thus facilitating appropriate changes in insulin therapy, is a major step towards improved diabetes monitoring. Several recent studies have shown a clinical benefit of CGM on reduction of glycated haemoglobin (HbAlc) ${ }^{7-10}$ and the burden of hypoglycaemia ${ }^{11}$ in those using the device at least 6 of 7 days. The combination of real-time CGM with insulin pump therapy (sensor augmented insulin pump therapy) with the ability to temporarily suspend insulin delivery at preset glucose levels (threshold based pump suspension) has also shown to reduce the burden of hypoglycaemia. ${ }^{13} 14$

The development of a closed-loop system that combines glucose monitoring with computer-based algorithm dictated insulin delivery, may provide further improvements in glycaemic control while reducing hypoglycaemia and represents a realistic treatment option for people with T1Ds. ${ }^{15}$ Closed-loop differs from conventional pump therapy, characterised by preprogrammed basal delivery, through the use of a control algorithm which directs subcutaneous insulin delivery according to sensor glucose levels. The role of the control algorithm is to translate, in real-time, the information it receives from the glucose sensor and to compute the amount of insulin to be delivered by the pump.

Several studies have evaluated the safety and efficacy of closed-loop under laboratory conditions and shown promising results. These include evaluations using a randomised design by our group in youths, ${ }^{16}{ }^{17}$ adults $^{18}$ and pregnant women ${ }^{19}$ and by others using the model predictive control algorithm, ${ }^{20}{ }^{21}$ the proportionalintegral-derivative approach $^{22} 23$ and the fuzzy logic controller. $^{24} 25$ Insulin and glucagon coadministration have also been evaluated. ${ }^{26-28}$

In contrast to studies conducted in the clinical research facility with carefully controlled conditions, closed-loop at home is exposed to considerably more varied meal and exercise patterns, and other life-style modifiers. Participants may overestimate or underestimate carbohydrate content and may undertake unplanned activity and/or exercise. People using insulin pump therapy are advised to use temporary reductions or increments of basal insulin delivery to meet these demands but this requires a degree of planning and user intuition and interaction. Since closed-loop systems modulate delivery of insulin in a glucose responsive fashion, ${ }^{15}$ they may be able to achieve better glucose control than preprogrammed basal rates of conventional pump therapy.

We have recently completed three short-term home closed-loop studies under free living conditions (table 1). Two studies evaluated overnight closed-loop glucose control in adolescents (3 weeks) and adults (4 weeks) and one study evaluated day and night closed-loop control in adults (1-week). During both overnight studies, closed-loop achieved significantly better glucose control than standard insulin pump therapy combined with real time CGM without increasing the risk of hypoglycaemia ${ }^{29}$ ${ }^{30}$ or negatively impacting quality of life or psychosocial functioning. ${ }^{31}{ }^{32}$ Encouragingly similar benefits were also seen during fully automated day and night closed-loop glucose control with better time in target during day and night time periods. ${ }^{33}$

Based on promising results from the above three studies we plan to assess the efficacy, safety, acceptability and utility of medium term (3 months) day and night home closed-loop glucose control in adults at the home setting compared with sensor augmented pump therapy.

\section{METHODS AND ANALYSIS \\ Overview}

The present study is an open-label, three-centre, multinational, randomised, two-period crossover study comparing automated closed-loop glucose control with sensor augmented insulin pump therapy. Study participants will be adults aged 18 years or over with T1D treated with insulin pump therapy. Two study

Table 1 Primary outcome of three home closed-loop studies

\begin{tabular}{|c|c|c|c|c|c|c|c|}
\hline \multirow[b]{2}{*}{ Study } & \multirow{2}{*}{$\begin{array}{l}\text { Duration of home } \\
\text { closed- loop (weeks) }\end{array}$} & \multirow{2}{*}{$\begin{array}{l}\text { Closed-loop } \\
\text { period }\end{array}$} & \multirow[b]{2}{*}{ Population } & \multicolumn{2}{|c|}{$\begin{array}{l}\text { Time spent }(\%) \text { in target } \\
\text { glucose }^{\star}\end{array}$} & \multirow[b]{2}{*}{ p Value } & \multirow[b]{2}{*}{ Reference } \\
\hline & & & & Closed loop & Open loop† & & \\
\hline APCam06 & 3 & Overnight & Adolescents & 64 (45-79) & 47 (18-70) & $<0.001$ & 29 \\
\hline Angela03 & 4 & Overnight & Adults & $52.6 \pm 10.6$ & $39.1 \pm 12.8$ & $<0.001$ & 30 \\
\hline AP@ home02 & 1 & Day and night & Adults & 75 (61-79) & $62(53-70)$ & 0.005 & 33 \\
\hline
\end{tabular}


intervention periods will past 3 months each with 46 weeks wash out period. The order of the two interventions will be random. The study will be coordinated from the Institute of Metabolic Science, University of Cambridge, UK. The study will aim for 30 completed participants (10 participants per centre) recruited from:

1. Addenbrooke's Hospital, Cambridge University Hospitals NHS Foundation Trust, Cambridge, UK.

2. Profil Institute, Neuss, Germany

3. Medical University of Graz, Graz, Austria

Recruitment will target up to 42 participants (14 participants per centre) to allow for dropouts. Methods of participants' recruitment will follow well-established practice at each centre. Written informed consent will be obtained from all participants before any study related activities.

\section{Inclusion criteria}

1. The participant has T1D as defined by WHO

2. The participant is 18 years of age or older

3. The participant will have been on an insulin pump for at least 6 months with good knowledge of insulin self-adjustment including carbohydrate counting

4. The participant is treated with one of the rapid acting insulin analogues (insulin Aspart, insulin Lispro or insulin Glulisine)

5. HbAlc $\geq 7.5 \% \quad(58 \mathrm{mmol} / \mathrm{mmol})$ and $\leq 10 \%$ $(86 \mathrm{mmol} / \mathrm{mmol})$ based on analysis from central laboratory or equivalent (only three participants are allowed between HbAlc $\geq 7.5 \% \quad(58 \mathrm{mmol} / \mathrm{mmol})$ and $\leq 8.0 \%$ ( $64 \mathrm{mmol} / \mathrm{mol})$ per centre $)$. Recruitment will aim for equal proportion of participants above and below HbAlc $8.5 \%$ (69 mmol/mmol).

6. The participant is willing to perform regular capillary blood glucose monitoring, with at least six measurements per day

7. The participant is willing to wear closed-loop system at home and at work place

8. The participant is willing to follow study specific instructions

9. The participant is willing to upload pump and CGM data at regular intervals

10. The participant is willing to restrict alcohol consumption to $\leq 2$ units per day throughout the study period

11. Female participants of childbearing age should be on effective contraception and must have a negative urine human chorionic gonadotrophin pregnancy test at screening. In addition in Germany, women of childbearing potential must use a highly effective method of birth control, which is defined as those which result in a low failure rate (ie, less than $1 \%$ per year) and must use two independent methods of contraception, for example, diaphragm and spermicide-coated condom.

\section{Exclusion criteria}

1. Non-T1D
2. People who are living alone

3. Any other physical or psychological disease or condition likely to interfere with the normal conduct of the study and interpretation of the study results

4. Current treatment with drugs known to have significant interference with glucose metabolism, such as systemic corticosteroids, as judged by the investigator

5. Known or suspected allergy against insulin

6. People with clinically significant nephropathy (eGFR $<45 \mathrm{~mL} / \mathrm{min}$ ), neuropathy or active retinopathy (defined as presence of maculopathy or more than background diabetic retinopathy changes) as judged by the investigator

7. Significantly reduced hypoglycaemia awareness (Gold score $\geq 4$ according to Geddes $e t a l^{84}$

8. More than one episode of severe hypoglycaemia as defined by American Diabetes Association ${ }^{35}$ in preceding 12 months (severe hypoglycaemia is defined as an event requiring assistance of another person to actively administer carbohydrates, glucagon or take other corrective actions including episodes of hypoglycaemia severe enough to cause unconsciousness, seizures or attendance at hospital)

9. Random C-peptide $>100 \mathrm{pmol} / \mathrm{L}$ with concomitant plasma glucose $>4 \mathrm{mmol} / \mathrm{L}(72 \mathrm{mg} / \mathrm{dL})$

10. Total daily insulin dose $\geq 2 \mathrm{IU} / \mathrm{kg} /$ day

11. Person is pregnant or breast feeding or planning pregnancy within next 10 months

12. Severe visual impairment

13. Severe hearing impairment

14. People using implanted internal pacemaker

15. Lack of reliable telephone facility for contact

16. People not proficient in English (UK) or German (Germany and Austria).

Reflecting local practice several additional exclusion criteria were applicable to German site. These included people with positive results on urine drug screen, positive alcohol breath test, positive hepatitis $\mathrm{B}$ surface antigen, anti-hepatitis $\mathrm{C}$ virus antibodies, anti-HIV 1 antibodies, anti-HIV 2 antibodies, people with significant skin conditions, documented allergy to medical adhesives and those with eating disorders.

\section{Study schedule}

The study will consist of up to 20 visits or preplanned telephone/email contacts. Two study intervention periods will past 3 months each with 4-6 weeks wash out period between them. The order of the two treatments will be random. The first $6 \mathrm{~h}$ of each treatment period will be conducted at the clinical research facility of the respective centre. The study flow chart is shown in figure 1. Key activities undertaken during each study visit are shown in table 2.

\section{Study training}

After enrolment, participants will be trained on the use of study insulin pump (Dana R Diabecare, Sooil, Seoul, South Korea) and CGM device (FreeStyle Navigator II, Abbott Diabetes Care, Alameda, California, USA) ${ }^{36}$ The 
Figure 1 Study flow chart.

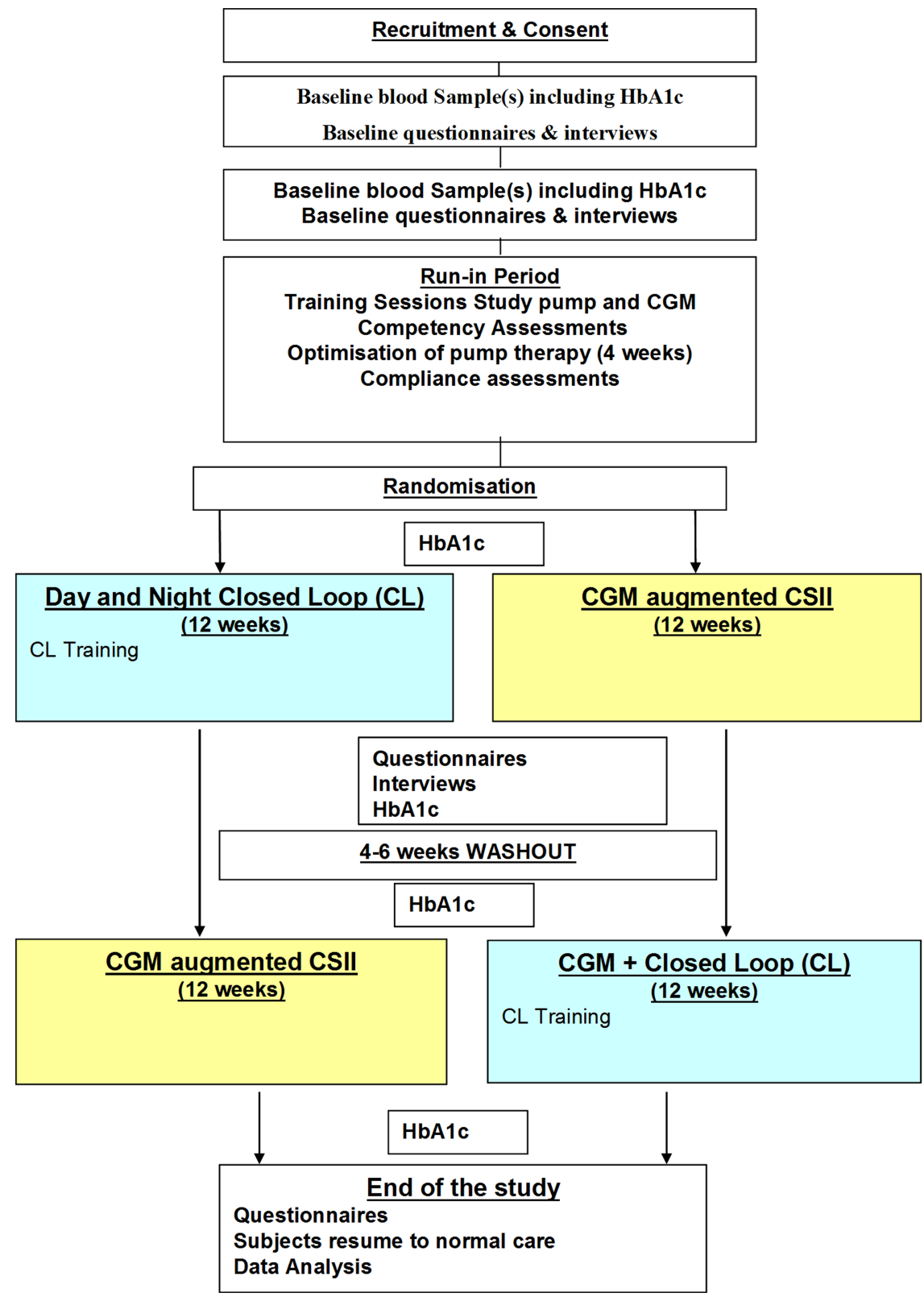

study insulin pump will be programmed with participant's usual basal settings as well as usual insulin to carbohydrate ratios and correction factors. Ability to use study devices will be formally assessed using competency assessment and additional training provided as required.

\section{Treatment optimisation period}

There will be a minimum of 4-week optimisation period for all participants. The participant will be invited to attend the research centre at weekly intervals during this period. The data from study insulin pump and CGM device will be downloaded and will be used for treatment optimisation. The visits will be conducted by a professional pump educator possibly accompanied by a member of the study team and will follow a written curriculum. During the optimisation period formal tests will be undertaken to assess the adequacy of basal and bolus setup of participants usual insulin pump therapy. At the end of the optimisation period participant's compliance of using the study CGM and study pump over preceding 14 days will be assessed. To proceed with the study participants need to demonstrate correct use of study insulin pump including the use of bolus calculator over $90 \%$ of meal boluses and at least 10 days' worth of CGM data during past 14 days of the optimisation period.

\section{Randomisation}

Eligible participants will be randomised to the order of the two study interventions using web-based permuted blocks of four randomisation based on computergenerated random code with study centre considered as a factor. Participants will be allocated as they are enrolled. 
Table 2 Schedule of study visits

\begin{tabular}{|c|c|c|}
\hline & Visit/contact & Description \\
\hline & Visit 1 & $\begin{array}{l}\text { Recruitment visit: consent, } \mathrm{HbA} 1 \mathrm{c} \text {, baseline bloods, urine pregnancy test, baseline } \\
\text { questionnaires and interviews }\end{array}$ \\
\hline \multirow[t]{2}{*}{ Training } & Visit 2 & Insulin pump training Initiation study pump, Competency assessment \\
\hline & Visit 3 & CGM training Initiation of study CGM, Competency assessment \\
\hline \multirow[t]{4}{*}{$\begin{array}{l}\text { Optimisation } \\
\text { ( } 4 \text { weeks) }\end{array}$} & Visit 4 & $\begin{array}{l}\text { Review pump and CGM data and optimisation of treatment. Further device training as } \\
\text { required }\end{array}$ \\
\hline & Visit 5 & Review pump and CGM data and optimisation of treatment \\
\hline & Visit 6 & Review pump and CGM data and optimisation of treatment \\
\hline & Visit 7 & Review pump and CGM data and optimisation of treatment \\
\hline \multirow[t]{8}{*}{$\begin{array}{l}\text { CL Intervention } \\
\text { (3 months) }\end{array}$} & Visit 8 & $\begin{array}{l}\mathrm{CL} \text { initiation at research facility, urine pregnancy test, } \mathrm{CL} \text { training, competency } \\
\text { assessment, } \mathrm{HbA1c}\end{array}$ \\
\hline & Visit $9^{*}$ & Review use of study devices; further optimisation as required \\
\hline & Visit $10^{*}$ & Review pump and CGM data; further optimisation as required \\
\hline & Visit $11^{*}$ & End of first month; review pump and CGM data; further optimisation as required \\
\hline & Visit $12^{*}$ & End of second month; review pump and CGM data; further optimisation as required \\
\hline & Visit 13 & $\begin{array}{l}\text { End of closed-loop treatment arm ( } 3 \text { months) } \mathrm{HbA1c} \text {. Complete questionnaires and } \\
\text { optional interviews. Collect algorithm device (optional return of CGM and study } \\
\text { pump) }\end{array}$ \\
\hline & - & Washout period \\
\hline & Visit 14 & Optional-collection of CGM and study pump if handed back during visit 13 \\
\hline \multirow[t]{6}{*}{$\begin{array}{l}\text { SAP Intervention } \\
\text { (3 months) }\end{array}$} & Visit 15 & $\begin{array}{l}\text { SAP initiation at research facility, urine pregnancy test, SAP training, competency } \\
\text { assessment, }-\mathrm{HbA} 1 \mathrm{c}\end{array}$ \\
\hline & Visit $16^{*}$ & Review use of study devices; further optimisation as required \\
\hline & Visit $17^{*}$ & Review pump and CGM data; further optimisation as required \\
\hline & Visit $18^{*}$ & End of first month; Review pump and CGM data; further optimisation as required \\
\hline & Visit 19* & End of second month; Review pump and CGM data; further optimisation as required \\
\hline & Visit 20 & $\begin{array}{l}\text { End of SAP arm ( } 3 \text { months) HbA1c. Complete questionnaires and optional interviews } \\
\text { Collect all study devices }\end{array}$ \\
\hline
\end{tabular}

\section{Home treatment period}

At the start of each treatment period participants will be briefly admitted to the clinical research facility in the morning. Over $1 \mathrm{~h}$ participants will be provided with relevant training required for safe and effective use of the study intervention. This will include training on connection and disconnection of the closed-loop system and switching between closed-loop and usual pump therapy. Particular attention will be paid to meal bolus procedure during treatment periods and the use of study devices during exercise. During closed-loop period the meal bolus procedure will include entering the amount of carbohydrates eaten and glucose level via the user interface of the computer algorithm device (smartphone). During sensor augmented pump therapy, participants will enter the information directly into the bolus calculator residing on insulin pump. The method of calculation of the meal bolus is identical between the two treatment periods and will utilise the bolus calculator of the study pump and will include a correction insulin dose for high or low glucose levels. Competency on the use of study devices will be assessed by the study team. Only participants who demonstrate competency on use of study devices will be allowed to continue to the home study phase. During this visit participants will have a blood test for HbA1c and will be asked to complete questionnaires as outlined below.

Participants will be allowed to go home at midafternoon. They will be advised to continue with study treatment at home over the next 3 months. Participants will be asked to download the data from the study pump and CGM device at regular intervals and will be allowed to drive while using study devices and adhering to usual precautions and country specific rules and regulations. Participants will be advised to discontinue automated closed-loop insulin delivery and follow their usual insulin pump therapy for periods of exercise in the first 2 weeks of closed-loop use. After first 2-week of use by participants who usually undertake exercise, closed-loop system may be applied during moderate exercise if practicable. During the first 2 weeks of each study period each participant will be advised against international travel. During the rest of study period travel inside European Economic Area will be allowed while closed-loop system is in use.

\section{Contact during 3 months home study period}

Participants will have identical planned contacts with the study team during the two treatment periods. This will include weekly for first 2 weeks and monthly for 
remainder of the study interventions. Except for the visits at the start and end of each study intervention, all other visits will be conducted via telephone/email. Participants will be provided with $24 \mathrm{~h}$ telephone helpline to contact the study team if they encounter any technical problems.

\section{Closed-loop system}

The FlorenceD2A closed-loop system (University of Cambridge, Cambridge, UK) ${ }^{37}$ comprises a model predictive control algorithm residing on a smartphone (Nexus 4, LG, South Korea), which communicates wirelessly with a purpose made translator unit (Triteq, Hungerford, UK) and the study pump through a Bluetooth communication protocol (figure 2). The CGM receiver is inserted into the translator which translates a serial USB protocol into a Bluetooth communication protocol and powers the CGM receiver when USB communication takes place. The translator uses rechargeable batteries to supply power.

Every $12 \mathrm{~min}$, the system calculates a new insulin infusion rate which is automatically sent to the study insulin pump. The calculations utilise a compartment model of glucose kinetics ${ }^{38}$ describing the effect of rapid-acting insulin analogues and the carbohydrate content of meals on glucose levels.

The control algorithm will be initialised using preprogrammed basal insulin delivery downloaded from the study pump. Additionally, information about participant's weight and total daily insulin dose will be entered at setup. During closed-loop operation, the algorithm adapts itself to a particular participant. The treat-to-target control algorithm aims to achieve glucose levels between 5.8 and $7.3 \mathrm{mmol} / \mathrm{L}$ and adjusts the actual level depending on fasting versus postprandial status and the accuracy of model-based glucose predictions.

The closed-loop system can operate on battery power over 2-4 days without recharging. The smartphone uploads data on a server using a $3 \mathrm{G} / \mathrm{GSM}$ communication to ease data transfer and downloads data from study devices during the sensor augmented pump therapy when the closed-loop control is disabled. The software residing on the smartphone comprises a bolus wizard, used during the closed-loop intervention to deliver meal and correction boluses, The bolus wizard comprises identical calculation procedures as bolus wizard residing on the study pump.

The CGM receiver provides hypoglycaemia and hyperglycaemia alarms, the insulin pump provides standard alarms, and the smartphone alerts the user about aspects related to closed-loop operation such as when closed loop started, stopped or terminated. The smartphone also visualises sensor glucose, insulin delivery, carbohydrate content and other relevant data.

\section{Safety precautions during closed loop}

Participants will be trained to perform a calibration check before breakfast and evening meal. If sensor
Figure 2 Design of Florence D2A automated closed-loop system.

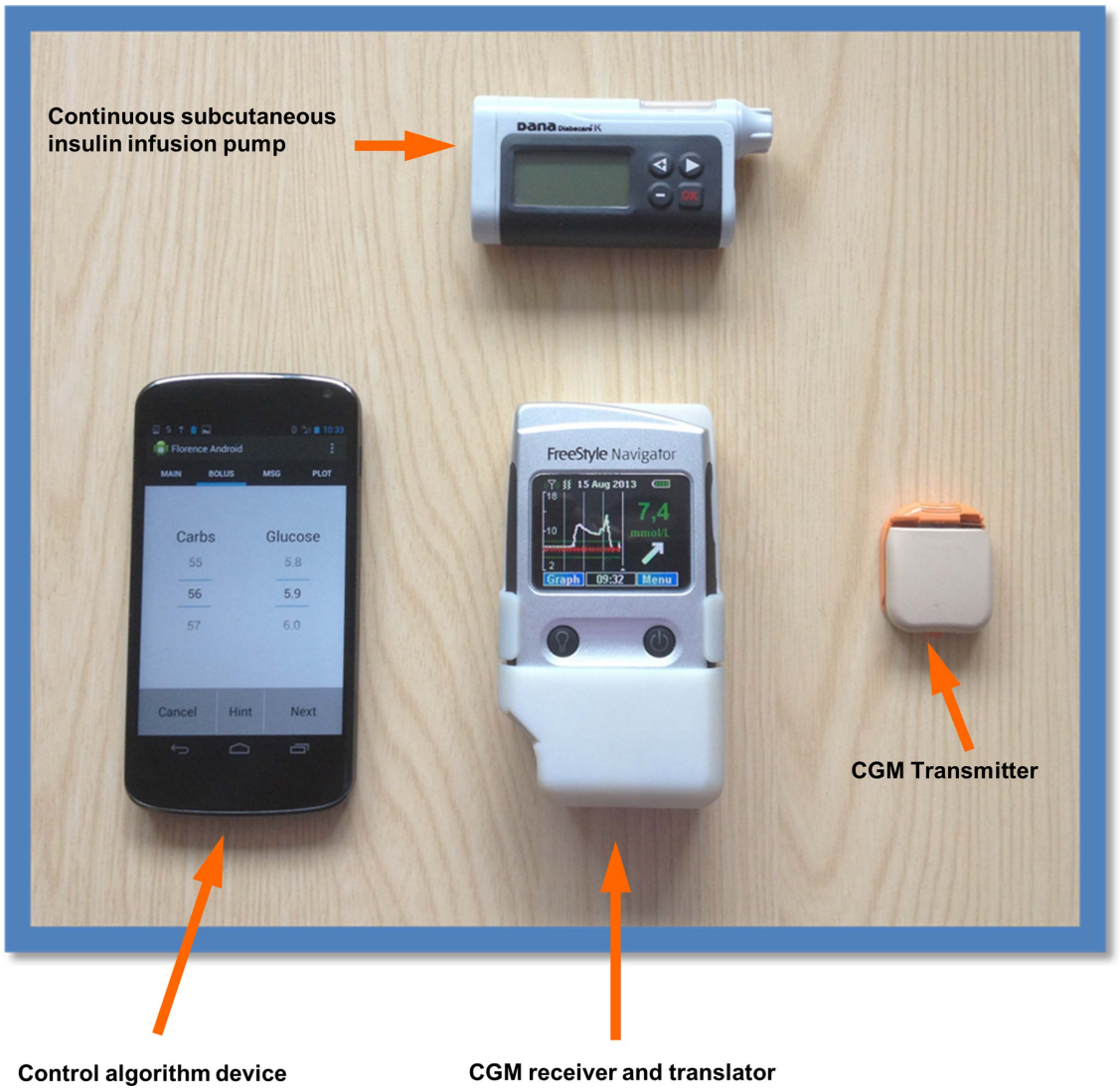


glucose is above finger-stick glucose by more than $3 \mathrm{mmol} / \mathrm{L}$, the CGM will be recalibrated. These instructions resulted from an in silico evaluations of hypoglycaemia and hyperglycaemia risk ${ }^{39}$ using the validated Cambridge simulator. ${ }^{40}$

If sensor glucose becomes unavailable, preprogrammed insulin delivery will be automatically restarted within 30 min or within $1 \mathrm{~h}$ in case of other failures. This limits the risk of insulin underdelivery and overdelivery. ${ }^{39}$ Safety rules limit maximum insulin infusion and suspend insulin delivery at sensor glucose at or $<4.3 \mathrm{mmol} / \mathrm{L}$ or when sensor glucose is rapidly decreasing.

\section{Participant withdrawal criteria}

The following withdrawal criteria will apply:

1. Participant is unable to demonstrate safe use of study insulin pump and/or CGM during run-in period as judged by the investigator.

2. Participant is unable to comply with study insulin pump and/or CGM during run-in period.

3. Participant may terminate participation in the study at any time without necessarily giving a reason and without any personal disadvantage.

4. Significant protocol violation or non-compliance.

5. Decision by the investigator or the sponsor that termination is in the participant's best medical interest

6. Participant becomes pregnant during the study period.

7. Allergic reaction to insulin.

8. If participant cannot be contacted in 4 weeks subject will be considered lost to follow-up.

\section{Questionnaires and interviews}

A mixed methods psychosocial evaluation using semistructured interviews and validated questionnaires, will be conducted to determine the utility of the device in terms of acceptability of intervention, quality of life, participants' perceptions of impact on lifestyle, diabetes selfmanagement and fear of hypoglycaemia.

\section{Qualitative methods}

Participants will be invited to join three semistructured telephone interviews conducted by a health psychologist. Interviews will explore participants' experiences, their expectations and feelings at baseline and then at the end of each phase that is, closed loop and sensor augmented pump therapy to determine whether the technologies matched expectations, whether any difficulties occurred, what were the benefits and downsides of living with the technologies for the duration of the trial and any another aspects that have arisen as a consequence of participation. These interviews will be broadly similar to interviews conducted in our previous closed-loop studies. ${ }^{31}$

\section{Quantitative methods}

Participants will be invited to complete two questionnaires, the Diabetes Technology Questionnaire and ADDQoL Questionnaire, at baseline and at the end of each study arm. The Diabetes Technology Questionnaire is a 30-item measure of the impact of, and satisfaction with, technological tools that may be used in the management of T1D. ${ }^{41}$ Participants will be asked to rate their agreement or disagreement with statements regarding the specific complement of diabetes technologies (ie, metre, pump, continuous glucose monitor and closed-loop system). Items related to 'current' satisfaction and impact as well as 'change' in satisfaction and impact since the new technology was added to the participant's regimen will be evaluated. The ADDQoL Questionnaire ${ }^{42}$ consists of two overview items; one measures generic overall quality of life and a further 19 items are concerned with the impact of diabetes on specific aspects of life. The 19 life domains are as follows: leisure activities, working life, local or longdistance journeys, holidays, physical health, family life, friendships and social life, close personal relationships, sex life, physical appearance, self-confidence, motivation to achieve things, people's reactions, feelings about the future, financial situation, living conditions, dependence on others, freedom to eat and freedom to drink. These 19 domains ask the respondents to evaluate how their life would be if they did not have diabetes.

\section{Statistical analysis}

All analysis will be conducted on an intention to treat basis. Data from all randomised participants with or without protocol violation including dropouts and withdrawals will be included in the analysis. The respective values obtained during the 3 months randomised interventions contrasting the closed-loop system against the sensor augmented pump therapy will be compared using a regression model that accounts for period effect. Residual values from the regression model will be examined for an approximate normal distribution. If values are highly skewed a transformation or nonparametric analyses will be used. Values will be given mean (SD) or as median (IQR) for each treatment (closed loop or sensor augmented pump therapy).

Selected CGM outcomes will also be calculated for (1) day time and (2) night time separately. The day time will be classified as between 08:00 and 23:00 h. The night time will be classified as between 23:00 until 08:00 $\mathrm{h}$. Safety analysis will include all available data from all recruited participants. A 5\% significance level will be used to declare statistical significance for all comparisons.

\section{The primary end point}

The primary outcome measure is time spent with CGM glucose concentration in the target range (3.9$10.0 \mathrm{mmol} / \mathrm{L}$ ) during 3 months home period. The primary analysis will be the single comparison of the above outcome between closed loop and control interventions and no attempt will be made to formally control the overall type I error rate for the secondary outcomes.

\section{The secondary end points}

The following outcomes on the two treatment arms will be compared: 
1. HbA1c

2. The area under the curve (AUC) below $3.5 \mathrm{mmol} / \mathrm{L}$ based on continuous subcutaneous glucose monitoring

3. Time spent with CGM glucose levels in hypoglycaemic range $(<3.9 \mathrm{mmol} / \mathrm{L})$.

4. Time spent with CGM glucose levels $<3.5 \mathrm{mmol} / \mathrm{L}$.

5. Time spent with CGM glucose levels $<2.8 \mathrm{mmol} / \mathrm{L}$.

6. Time spent with CGM glucose levels in hyperglycaemic range $(>10.0 \mathrm{mmol} / \mathrm{L})$.

7. Time spent with CGM glucose levels in significant hyperglycaemic range $(>16.7 \mathrm{mmol} / \mathrm{L})$.

8. Mean of CGM glucose levels.

9. 9. Coefficient of variation (CV) of CGM glucose levels.

10. Low Blood Glucose Index (LBGI) using CGM.

11. Weekly trend in glucose control and insulin delivery

12. Number of nights when sensor glucose was below $3.5 \mathrm{mmol} / \mathrm{L}$ for at least $20 \mathrm{~min}$

13. 13. Duration of periods when sensor glucose values was below $3.5 \mathrm{mmol} / \mathrm{L}$ for at least $20 \mathrm{~min}$

14. Total daily insulin dose

15. Total daily bolus insulin dose

16. Total daily basal insulin dose

17. CV of basal insulin delivery

18. Between $24 \mathrm{~h}$ period variability: $\mathrm{CV}$ of CGM glucose between $24 \mathrm{~h}$ periods (midnight to midnight)

19. Glucose concentration in the target range (3.9$10.0 \mathrm{mmol} / \mathrm{L})$, and above and below target range based on adjusted CGM. ${ }^{43}$

20. Psychosocial functioning

21. Quality of life

During overnight period between 23:00 and 08:00 (9 h)

the following outcomes will be calculated:

- Time spent with CGM glucose concentration in the target range $(3.9-8.0 \mathrm{mmol} / \mathrm{L})$

- Mean CGM glucose levels

- The AUC below $3.5 \mathrm{mmol} / \mathrm{L}$ based on continuous subcutaneous glucose monitoring

- CV of CGM glucose levels

- Between night variability: CV of CGM glucose between nights

- Total insulin dose.

During day period between 08:00 to 23:00 (15 h) the following outcomes will be calculated:

- Time spent with CGM glucose concentration in the target range $(3.9-10.0 \mathrm{mmol} / \mathrm{L})$

- Mean CGM glucose levels

- The AUC below $3.5 \mathrm{mmol} / \mathrm{L}$ based on continuous subcutaneous glucose monitoring

- CV of CGM glucose levels

- Between day-time variability: CV of CGM glucose between days

- Total insulin dose

\section{Safety analysis}

Safety data including number of participants and number of occurrences of severe hypoglycaemia events and significant ketonaemia $(>3.0 \mathrm{mmol} / \mathrm{L})$ with hyperglycaemia as well as nature and severity of any other adverse events (AEs) including serious adverse device effects (SADEs) and serious AEs (SAEs) will be tabulated for all subjects, including dropouts and withdrawals, irrespective of whether CGM data are available and irrespective of whether closed loop was operational.

\section{Utility outcomes during home phase}

System performance will be assessed by evaluating:

- Total number of hours and percentage of closed-loop operation

- Total number of hours and percentage of CGM availability

- Time between failures (total hours of closed-loop operation over number of failures (after closed-loop started))

- Time between failures due to CGM unavailability (total hours of closed-loop operation over number of terminating conditions due to CGM unavailability after closed loop started)

- Time between failures due to pump connectivity (total hours of closed-loop operation over number of terminating conditions due to pump connectivity (after closed loop started))

- Time between other failures (total hours of closed-loop operation over number of terminating conditions due to other reasons (after closed loop started)).

\section{CGM accuracy at home}

CGM accuracy during 3-month home phase against capillary glucose measurements will be evaluated using standard measures of numerical and clinical accuracy including mean and median absolute relative deviation, bias, International Standardization Organization criteria and Clarke error grid analysis. ${ }^{44}$

\section{Exploratory analysis}

Per protocol analysis will be conducted to explore the relationship between usage of study treatments and study outcomes. Additional analysis will include assessment of study outcomes on weekly/monthly basis, and contrasting night versus waking hour outcomes and those with HbA1c above and below $8.5 \%$ (69 mmol/ mol). Glucose control during the optimisation period will also be evaluated.

\section{Interim analysis}

No interim analysis will be performed.

\section{Power calculation}

Power calculation was based on the anticipated improvements the primary outcome, the time in spent in the target glucose range $(3.9-10.0 \mathrm{mmol} / \mathrm{L})$ during 3 months home stay. Assuming a SD of $18 \%$ at baseline and average improvement of time in target of 10\%, 31 participants are required at the desired $80 \%$ power and an $\alpha$ level of 0.05 (two-tailed). ${ }^{45}$ Above assumptions are in line with results from our 1-week home closed-loop 
feasibility study. ${ }^{33}$ To allow for dropouts up to 42 participants may be recruited aiming for 30 completed participants. Participants who dropout of the study during optimisation period and within the first 4 weeks of the first intervention will be replaced.

\section{STUDY MANAGEMENT}

\section{Data monitoring and ethics committee}

An independent Data Monitoring and Ethics Committee (DMEC) will comprise a chairperson and two experts. The DMEC will be informed of all SAEs and any unanticipated adverse device effects/events that occur during the study. The DMEC will review compiled $\mathrm{AE}$ data at periodic intervals. The DMEC will report to the Study Management Committee any safety concerns and recommendations for suspension or early termination of the investigation. Composition of the DMEC is shown in online supplementary appendix 1 .

\section{Study management committee}

A study management committee consisting of the Chief Investigator, Study Coordinator, and Study Data Manager will meet quarterly to discuss the operational aspects of the study. The Principal Clinical Investigators may also participate. Composition of the study management team is shown in online supplementary appendix 1.

\section{Study monitoring}

The Study Coordinator will ensure that the study is conducted in accordance with ICH GCP standards through site monitoring visits. A monitoring plan will be written and agreed prior to randomisation.

\section{Data management}

Confidentiality of participant data shall be observed at all times during the study. Personal details for each participant taking part in the research study and linking them to a unique identification number will be held locally on a study screening $\log$ in the Trial Master File at each of the investigation centres. These details will not be revealed at any other stage during the study, and all results will remain anonymous. The study identification number will be used on the case report forms and on all the blood and serum samples that are collected throughout the study. Names and addresses will not be used. Collected samples will be stored securely and locked away. Only researchers directly involved in the study will have access to the samples.

Electronic data will be stored on password-protected computers. All paper records will be kept in locked filing cabinets, in a secure office at each of the investigation centres. Only members of the research team and collaborating institutions will have password access to the anonymised electronic data. Only members of the research teams will have access to the filing cabinet. Paper copies of the data will be stored for 15 years in line with the Data Protection Act 1998.
Direct access to the source data will be provided for monitoring, audits, ethical committee review and regulatory authority inspections during and after the study. The fully anonymised data may be shared with third parties (EU or non-EU based) for the purposes of advancing management and treatment of diabetes.

Appropriate procedures agreed by the Chief Investigator and Clinical Principal Investigators will be put in place for data review, database cleaning and issuing and resolving data queries.

\section{Indemnity}

Indemnity for any harm arising from the conduct of research will be provided according to local arrangements in respective centre.

- Cambridge, UK-National Health Service indemnity cover will apply for any claims arising from management and conduct of research. Any liability arising from study design will be covered by the clinical trial insurance policy organised by the University of Cambridge.

- Neuss, Germany-Profil Institute for Metabolic Research, Neuss, Germany, will provide insurance for the participants according to local requirements.

- Graz, Austria-Participants will be insured according to Medical Device Law $\S 47$ (StF: BGBl. Nr. 657/ 1996, BGBl. I Nr. 143/2009)

\section{ETHICS AND DISSEMINATION}

The study has received approval from independent research ethics committees in UK, Germany and Austria. In addition the study has undergone a review by regulatory authorities in the UK (MHRA), Germany (BfArM) and Austria (AGES). All participants will be provided with oral and written information about the trial, including the most common AEs, and the procedures involved in the study before obtaining written informed consent. The study will be jointly sponsored by the University of Cambridge and the Cambridge University Hospitals NHS Foundation Trust, Cambridge, UK. Standard operating procedures for monitoring and reporting of all AEs and adverse device events will be in place, including SAE, SADE and specific AEs such as severe hypoglycaemia. The DMEC will be informed of all serious AEs and any unanticipated adverse device/method effects that occur during the study and will review compiled $\mathrm{AE}$ data at periodic intervals.

It is expected that screening and recruitment will commence in April/May 2014 and the study will be completed by summer 2015. The study results will be disseminated by peer-review publications and conference presentations.

\section{Author affiliations}

${ }^{1}$ Wellcome Trust-MRC Institute of Metabolic Science, University of Cambridge, Cambridge, UK

${ }^{2}$ Profil Institut für Stoffwechselforschung GmbH, Neuss, Germany 
${ }^{3}$ Division of Endocrinology and Metabolism, Department of Internal Medicine, Medical University of Graz, Graz, Austria

${ }^{4}$ Faculty of Medicine, Department of Human Development and Health, University of Southampton, Southampton, UK

${ }^{5}$ Center for Health Care Delivery Science, Nemours Children's Health System, Florida, USA

Acknowledgements Jasdip Mangat supported development and validation of closed-Ioop system. Josephine Hayes (Institute of Metabolic Science, University of Cambridge) provided administrative support.

Contributors LL, SD, JKM, CB, ME, LH, HK, HT, MEW, TRP, SA, MLE and RH codesigned the study. KB and TW were responsible designing the psychosocial assessments. RH designed and implemented the glucose controller. $\mathrm{LL}$ and $\mathrm{RH}$ wrote the manuscript. All authors critically reviewed the report.

Funding Seventh Framework Programme of the European Union (Grant Agreement number 247138), NIHR Cambridge Biomedical Research Centre, and Juvenile Diabetes Research Foundation.

Competing interests $\mathrm{RH}$ reports having received speaker honoraria from Minimed Medtronic, Lifescan, Eli Lilly, BBraun and Novo Nordisk, serving on advisory panel for Animas, Minimed Medtronic, and Eli Lilly, receiving license fees from BBraun and Beckton Dickinson; and having served as a consultant to Beckton Dickinson, BBraun, Sanofi and Profil. MLE reports having received speaker honoraria from Abbott Diabetes Care, Animas, serving on advisory board for Medtronic, Roche, Cellnovo. MEW has received license fees from Becton Dickinson and has served as a consultant to Beckton Dickinson. RH and MEW report patent applications. LH is partner and consultant of Profil Institut für Stoffwechselforschung, Neuss, Germany and Profil Institute for Clinical Research, San Diego, USA. He is a consultant for a number of companies that are developing novel diagnostic and therapeutic options. JKM reports having received speaker honoraria from NovoNordisk A/S. ME is employed by B. Braun Melsungen AG. TRP reports having received speaker honoraria from Novo Nordisk and Roche Diagnostics, serving on advisory panel for Novo Nordisk, BMS/Astra Zeneca, and Roche Diagnostics. KB reports having received speak honoraria from NovoNordisk A/S, Roche Diagnostics, Lifescan, Sanofi, Animas and Janssen. She is a member of the Roche Global Advisory Board, Roche European Insulin Delivery Systems Advisory Board and Animas CHOICE advisory board

Ethics approval Cambridge Central Research Ethics Commitee.

Provenance and peer review Not commissioned; internally peer reviewed.

Open Access This is an Open Access article distributed in accordance with the Creative Commons Attribution Non Commercial (CC BY-NC 4.0) license, which permits others to distribute, remix, adapt, build upon this work noncommercially, and license their derivative works on different terms, provided the original work is properly cited and the use is non-commercial. See: http:// creativecommons.org/licenses/by-nc/4.0/

\section{REFERENCES}

1. International Diabetes Federation. The diabetes atlas. 4th edn Brussels, 2009.

2. DIAMOND Project Group. Incidence and trends of childhood Type 1 diabetes worldwide 1990-1999. Diabet Med 2006;23:857-66.

3. Patterson CC, Dahquist GG, Gyurus E, et al. Incidence trends for childhood type 1 diabetes in Europe during 1989-2003 and predicted new cases 2005-20: a multicentre prospective registration study. Lancet 2009;373:2027-33.

4. Cryer PE. Elimination of hypoglycemia from the lives of people affected by diabetes. Diabetes 2011:60:24-7.

5. De Beaufort CE, Swift PG, Skinner CT, et al:; Hvidoere Study Group on Childhood Diabetes 2005. Continuing stability of center differences in pediatric diabetes care: do advances in diabetes treatment improve outcome? The Hvidoere Study Group on Childhood Diabetes. Diabetes Care 2007;30:2245-50.

6. Hoerger TJ, Segel JE, Gregg EW, et al. Is glycemic control improving in U.S. adults? Diabetes Care 2008;31:81-6.

7. Deis D, Bolinder J, Riveline JP, et al. Improved glycemic control in poorly controlled patients with type 1 diabetes using real-time continuous glucose monitoring. Diabetes Care 2006;29:2730-2.
8. Tamborlane WV, Beck RW, Bode BW, et al:; Juvenile Diabetes Research Foundation Continuous Glucose Monitoring Study Group. Continuous glucose monitoring and intensive treatment of type 1 diabetes. N Engl J Med 2008;359:1464-76.

9. Kordonouri O, Pankowska E, Rami B, et al. Sensor-augmented pump therapy from the diagnosis of childhood type 1 diabetes: results of the Paediatric Onset Study (ONSET) after 12 months of treatment. Diabetologia 2010;53:2487-95.

10. Hirsch IB, Abelseth J, Bode BW, et al. Sensor-augmented insulin pump therapy: results of the first randomized treat-to-target study. Diabetes Technol Ther 2008;10:377-83.

11. Battelino T, Phillip M, Bratina N, et al. Effect of continuous glucose monitoring on hypoglycemia in type 1 diabetes. Diabetes Care 2011;34:795-800.

12. Choudhary P, Ramasamy S, Green L, et al. Real-time continuous glucose monitoring significantly reduces severe hypoglycemia in hypoglycemia-unaware patients with type 1 diabetes. Diabetes Care 2013;36:4160-2.

13. Bergenstal RM, Klonoff DC, Garg SK, et al:; ASPIRE In-Home Study Group. Threshold-based insulin-pump interruption for reduction of hypoglycemia. N Engl J Med 2013;369:224-32.

14. Ly TT, Nicholas JA, Retterath A, et al. Effect of sensor-augmented insulin pump therapy and automated insulin suspension vs standard insulin pump therapy on hypoglycemia in patients with type 1 diabetes: a randomized clinical trial. JAMA 2013;310:1240-7.

15. Hovorka R. Closed-loop insulin delivery: from bench to clinical practice. Nat Rev Endocrinol 2011;7:385-95.

16. Hovorka R, Allen JM, Elleri D, et al. Manual closed-loop insulin delivery in children and adolescents with type 1 diabetes: a phase 2 randomised crossover trial. Lancet 2010;375:743-51.

17. Elleri D, Allen JM, Kumareswaran K, et al. Closed-loop basal insulin delivery over 36 hours in adolescents with type 1 diabetes: randomized clinical trial. Diabetes Care 2013:36:838-44.

18. Hovorka R, Kumareswaran K, Harris J, et al. Overnight closed loop insulin delivery (artificial pancreas) in adults with type 1 diabetes: crossover randomised controlled studies. BMJ 2011;342:d1855.

19. Murphy HR, Kumareswaran K, Elleri D, et al. Safety and efficacy of 24-h closed-loop insulin delivery in well-controlled pregnant women with type 1 diabetes: a randomized crossover case series. Diabetes Care 2011;34:2527-9.

20. Kovatchev B, Cobelli C, Renard E, et al. Multinational study of subcutaneous model-predictive closed-loop control in type 1 diabetes mellitus: summary of the results. J Diabetes Sci Technol 2010;4:1374-81.

21. Breton M, Farret A, Bruttomesso D, et al.; International Artificial Pancreas Study Group. Fully integrated artificial pancreas in type 1 diabetes: modular closed-loop glucose control maintains near normoglycemia. Diabetes 2012;61:2230-7.

22. Dauber A, Corcia L, Safer J, et al. Closed-loop insulin therapy improves glycemic control in children aged \&lt;7 years: a randomized controlled trial. Diabetes Care 2013;36:222-7.

23. Sherr JL, Cengiz E, Palerm CC, et al. Reduced hypoglycemia and increased time in target using closed-loop insulin delivery during nights with or without antecedent afternoon exercise in type 1 diabetes. Diabetes Care 2013;36:2909-14.

24. Atlas E, Nimri R, Miller S, et al. MD-Logic Artificial Pancreas System A pilot study in adults with type 1 diabetes. Diabetes Care 2010;33:1072-6.

25. Nimri R, Danne T, Kordonouri O, et al. The "Glucositter" overnight automated closed loop system for type 1 diabetes: a randomized crossover trial. Pediatr Diabetes 2013;14:159-67.

26. Haidar A, Legault L, Dallaire M, et al. Glucose-responsive insulin and glucagon delivery (dual-hormone artificial pancreas) in adults with type 1 diabetes: a randomized crossover controlled trial. CMAJ 2013;185:297-305.

27. Castle JR, Engle JM, El Youssef J, et al. Novel use of glucagon in a closed-loop system for prevention of hypoglycemia in type 1 diabetes. Diabetes Care 2010;33:1282-7.

28. Russell SJ, El-Khatib FH, Nathan DM, et al. Blood glucose control in type 1 diabetes with a bihormonal bionic endocrine pancreas. Diabetes Care 2012;35:2148-55.

29. Hovorka R, Elleri D, Thabit $\mathrm{H}$, et al. Overnight closed-loop insulin delivery in young people with type 1 diabetes: a free-living, randomized clinical trial. Diabetes Care 2014;37:1204-11.

30. Thabit H, Lubina-Solomon A, Stadler M, et al. Home use of closed-loop insulin delivery for overnight glucose control in adults with type 1 diabetes: a 4-week, multicentre, randomised crossover study. Lancet Diabetes Endocrinol Published Online First: 16 Jun 2014.

31. Barnard KD, Wysocki T, Allen JM, et al. Closing the loop overnight at home setting: psychosocial impact for adolescents with type 1 
diabetes and their parents. BMJ Open Diab Res Care 2014;2: e000025.

32. Barnard K, Thabit H, Lubina-Solomon A, et al. Overnight closed-loop insulin delivery: psychosocial impact for adults with type 1 diabetes. Diabetes 2014;63:A198.

33. Leelarathna L, Dellweg S, Mader JK et al: AP@ home Consortium. Day and night home closed-loop insulin delivery in adults with type 1 diabetes: three-center randomized crossover study. Diabetes Care 2014:37:1931-7.

34. Geddes J, Wright RJ, Zammitt NN, et al. An evaluation of methods of assessing impaired awareness of hypoglycemia in type 1 diabetes. Diabetes Care 2007;30:1868-70.

35. Seaquist ER, Anderson J, Childs B, et al. Hypoglycemia and diabetes: a report of a workgroup of the American Diabetes Association and the Endocrine Society. Diabetes Care 2013;36:1384-95.

36. Geoffrey M, Brazg R, Richard W. FreeStyle Navigator Continuous Glucose Monitoring System with TRUstart algorithm, a 1-hour warm-up time. J Diabetes Sci Technol 2011;5:99-106.

37. Elleri D, Allen JM, Biagioni M, et al. Evaluation of a portable ambulatory prototype for automated overnight closed-loop insulin delivery in young people with type 1 diabetes. Pediatr Diabetes 2012:13:449-53.

38. Hovorka R, Shojaee-Moradie F, Carroll PV, et al. Partitioning glucose distribution/transport, disposal, and endogenous production during IVGTT. Am J Physiol Endocrinol Metab 2002;282:E992-1007.
39. Wilinska ME, Budiman ES, Taub MB, et al. Overnight closed-loop insulin delivery with model predictive control: assessment of hypoglycemia and hyperglycemia risk using simulation studies. $J$ Diabetes Sci Technol 2009;3:1109-20.

40. Wilinska ME, Chassin LJ, Acerini CL, et al. Simulation environment to evaluate closed-loop insulin delivery systems in type 1 diabetes. J Diabetes Sci Technol 2010;4:132-44.

41. Juvenile Diabetes Research Foundation Continuous Glucose Monitoring Study Group. Validation of measures of satisfaction with and impact of continuous and conventional glucose monitoring Diabetes Technol Ther 2010;12:679-84.

42. Bradley C, Todd C, Gorton T, et al. The development of an individualized questionnaire measure of perceived impact of diabetes on quality of life: the ADDQoL. Qual Life Res 1999;8:79-91.

43. Hovorka R, Nodale M, Haidar A, et al. Assessing performance of closed-loop insulin delivery systems by continuous glucose monitoring: drawbacks and way forward. Diabetes Technol Ther 2013;15:4-12.

44. Clarke W, Kovatchev B. Statistical tools to analyze continuous glucose monitor data. Diabetes Technol Ther 2009;11(Suppl 1): S45-54.

45. Norman G, Monteiro S, Salama S. Sample size calculations: should the emperor's clothes be off the peg or made to measure? BMJ 2012;345:e5278. 